\title{
Scaling Effectiveness in Existing Research-based Scheduling Practices in LTE-based Network
}

\author{
Srinivasa R. K. \\ Research Scholar \\ Visvesvaraya Technological University, \\ Department of Electronics \& Communication \\ Engineering, BIT, Bangalore, India
}

\author{
Hemantha Kumar A. R., PhD \\ Professor and Principal Investigator (R\&D) \\ Department of Electronics \& Communication \\ Engineering, BIT, Bangalore, India
}

\begin{abstract}
With the increasing demands of the communication performance over mobile networks, Long-Term Evolution has been observed to offer a significant contributory role by facilitating higher data transmission rates to its dynamic users. One of the core operations in the LTE-based network is scheduling which directly governs the part of communication in the presence of significant, small, medium traffic. Although there are beneficial advantages of LTE towards its catering up network demands, it too encounters some potential and unreported problems in the presence of large truck with the uncertainty of its associated load. Hence, the role of scheduling becomes most essential in this regards. This paper reviews some of the signatory work carried out in recent time and explored their contribution by measure the score of advantages as well as limitation associated with it. The paper also contributes by providing the crisp points that have been identified as the open research issues in the shape of research gap towards scheduling techniques in LTE.
\end{abstract}

\section{Keywords}

Long-Term Evolution (LTE); 4G; Scheduling; Uplink Transmission; Downlink Transmission

\section{INTRODUCTION}

The objective of Long-Term Evolution (LTE) is mainly to cater up the exponentially increasing demands of the dynamic users of the cellular network [1]. LTE is meant for offering a maximized rate of transmission with much-reduced network delay and reduced operational cost [2]. The primary goal of the scheduling process in LTE is essentially meant for offering a proper allocation of the resource to assist the variable demands of resources during data transmission process [3]. At present, the scheduling process is designed considering the explicit condition of the communication channel, the volume of traffic, and quality of service [4]. A good scheduler will have potential dependencies on gain factor involved in channel state associated with each user as well as sub-carriers. To offer lower delay in the scheduling process, the standards of LTE claims of providing one millisecond of duration involved in data forwarding process [5]. Important information in this regards is that such scheduling process is always considered to be carried out over base station. While executing the scheduling decision, the base station performs allocation of resources considering both downlinks as well as uplink transmission [6][7]. At present, LTE uses Orthogonal Frequency Division Multiplexing (OFDM) or Orthogonal Frequency Division Multiplexing Access OFDMA for performing uplink transmission while the downlink transmission is assisted by Single-Carrier Frequency Division Multiple Access (SC-FDMA). It is interesting to know that mechanism of scheduling in LTE is very much different from another form of technologies of accessing, where LTE based programming is carried out considering both time and frequency domain for users while other access technologies are limited to only time domain [8]. The problem associated with the scheduling process in LTE is all about identifying the precise allocation of scheduling blocks to specific user equipment's to increase cumulative throughput or minimizing network delay [9]. In order to avoid any issues, the present research work towards schedulers is designed to be functional in two, i.e. scheduling packet using time domain first and then execute frequency domain [10]-[20]. There also existing sure research work that uses multiple-input multiple-output as a scheme to design scheduler for LTE networks [21][22]. There have been various work carried out towards LTE network; however; multiple problems and issues are still demanding more investigation. For example, existing research work is not found to report any form of long supportability of spatial multiplexing during both uplink and downlink transmission for more number of layers to increase the transmission rates. There is also a significant problem associated with both the uplink and downlink transmission when they were made to work in multipoint forwarding and receiving of packets for increasing the throughput and coverage of extended rate of data. It is also found that adoption of relaying methodologies towards improving the optimal usage of the communication channel as well as its associated capacity has been less explored. All these problems are unsolved and unaddressed and require a good series of dedicated investigation in order to investigate a better form of the solution.

Hence, this paper reviews the existing approaches towards scheduling process in the LTE-based network and deliberately attempts to identify the potential research gap. The idea is to assist the upcoming researchers to measure the level of effectiveness in existing scheduling techniques. The organization of this paper is as follows: Section 2 discusses the theoretical briefing of LTE followed by an exhaustive review of current scheduling practices in Section 3. Section 4 discusses research gap while Section 5 briefs about the contribution of this paper followed by tentative future work direction.

\section{ABOUT LONG-TERM EVOLUTION}

LTE stands for Long Term Evolution invented by 3GPP (Third Generation Partnership Project) organization. LTE is a $4 \mathrm{G}$ wireless broadband service for Global System for mobile communications that are GSM and CDMA. The evolution of LTE comes from the previous telecom service known as UMTS (Universal Mobile Telecommunication System). Fig.1 highlights the conventional reference mode used in the study and analysis of the LTE-based network. LTE service provides high data rates, reduced latency, and is compatible with traditional GSM and 
UMTS technologies. LTE includes internet services with great potential data rates that are $300 \mathrm{Mbps}$ peak downlink and 75 Mbps peak uplink. LTE technology assists multiple data services such as voice, video and messaging traffic. LTE utilizes Orthogonal Frequency Division Multiplexing (OFDM) is used in downlink and Signal-carrier frequency division multiple access (SD-FDMA) is used in uplink. Signal to noise ratio (SNR) and Multiple Input Multiple Output (MIMO) technology which enable to provide efficient coverage and throughput. LTE technology also uses two duplex mode Frequency division duplex (FDD) and Time division duplex (TDD) for splitting the transmission direction for the user and network station. In FDD the uplink and downlink are broadcast with using different frequencies and in TDD the in wireless communications that use a single radio frequency to transmit in uplink and downlink directions. The LTE reference models discuss below which contains LTE entities and EPC entities, and it has involved two components that access network and core network.

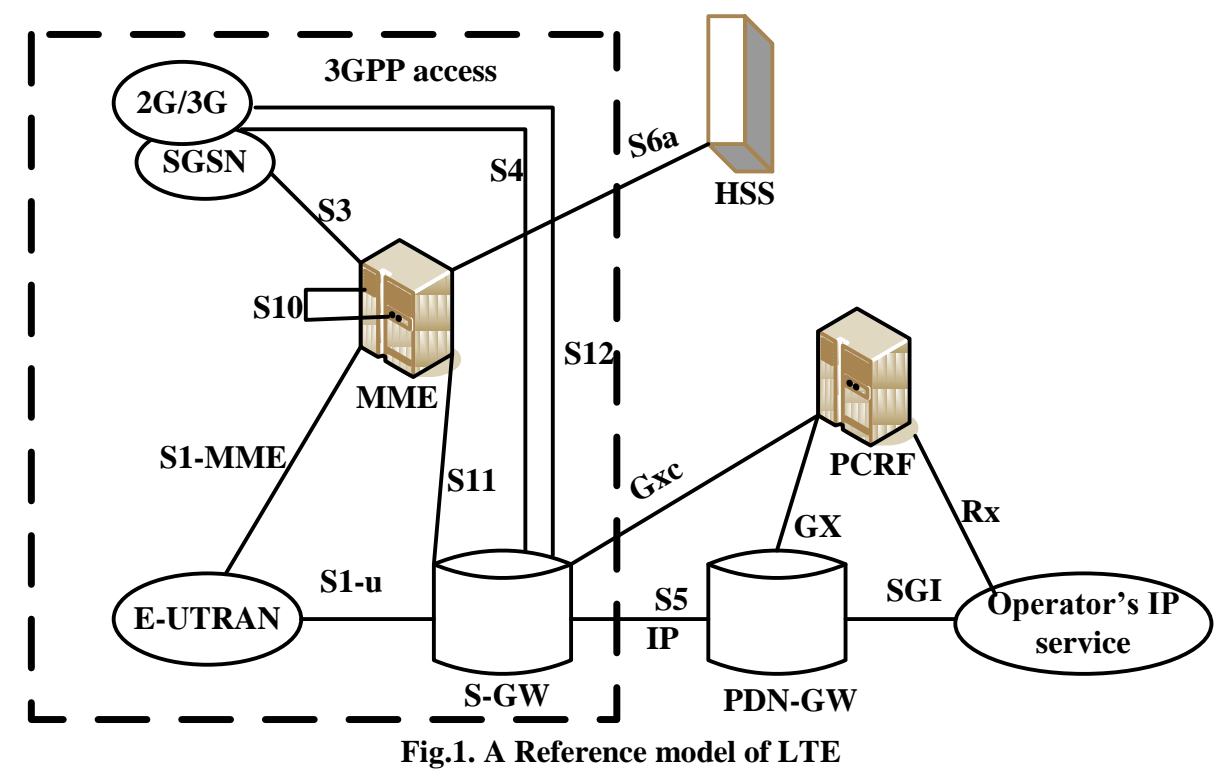

The access network contains E-UTRAN (Universal Terrestrial Radio Access Network). The core network comprises Evolved Packet Core (EPC) which is constructed to support an-3Gpp access support for mobile IPs. EPC is also responsible for network control for holding user subscription details which are addressed by HSS (Home Subscriber Server). MME (Mobility management entity) is the main control for the E-UTRAN which supports NAS signaling, Mobility management, User authentication and roaming with HSS. S-GW (Serving gateway) used to terminate the interface among E-UTRAN. PDN-GW (Packet Data Network Gateway) is responsible for IP routing and forwarding, UE IP address allocation. The massive increasing mobile data usage, the demand of the new application, online data communication, data streaming and need of real-time use and requirement of various global organizations which attracts huge attention regarding getting continual flow, reliable and efficient data transmission through internet services. So LTE service is introduced to full-fill all these requirements and offers various benefits to the global sector. The main objective of LTE is to provide a high data transmission rate, low latency and packet optimized radio access technology supporting flexible bandwidth deployments. The essential characteristics of LTE are as follows:

- Network selection and Detection- a mobile terminal that uses the features of various radio technologies or softwaredefined radios for permits contributions of multiple networks simultaneously, therefore, achieves optimal communication network with suitable service parameters.

- Consistent handover and service- a base station which is featured by inter and intra-technology handovers, that provide service stability with least disruption.
- LTE uses Orthogonal Frequency Division Multiplexing Access (OFDMA) that allows distributing multiple channels into groups depends upon the needs of each user.

- Increased Spectrum flexibility.

- Supports partially 200 active data clients in each 5 MHZ cell.

- $\quad$ Packet switched interface.

The adoption of LTE is associated with following beneficial characteristics

- Performance- LTE provides high-speed data transmission and better signal coverage.

- $\quad$ Simplicity- LTE supports scalable radio frequencies and uses two duplex technologies (FDD and TDD).

- Flexibility - LTE supports various features that used in advance media system or high-end-devices and such as online streaming, plug, and play, remote control systems, etc.

- Global sector- LTE used in various organizations and used in wide range transmission such as it provides reliable and long-term transmission in real time system and it allows giving internet facility on multiple devices.

- $\quad$ Supporting the consumer and operator requirements - LTE meets the needs of customer and operators. In case of a customer, it gets high speed and flexible data services at efficient variable cost. In fact of an operator, they are active in developing new features and using of Flexible radio spectrum that is $1.4 \mathrm{MHZ}$ to $20 \mathrm{MHZ}$ channels providing 
various feature plans to their consumer which in result more customers are connected with them.

- Simple architecture and open interfaces

- Flexible use of existing and new frequency bands

However, apart from the above stated beneficial characteristics, LTE scores well in performance in contrast to existing networks as:

- Improved speed and latency.

- Supports real-time-applications due to its low latency

- Offers great potential data transmission rate

- Offers great security and privacy

- Supports global systems with implicit mobility.

- Great end-user experience

- $\quad$ Reduce cost per bit

Table 1 highlights the conventional parameters involved in the study of LTE-based networks.

Table.1. Basic parameters of LTE

\begin{tabular}{|c|c|}
\hline Parameters & Description \\
\hline Duplexing & $\begin{array}{l}\text { Frequency division duplex, } \\
\text { time division duplex, half } \\
\text { duplex frequency division } \\
\text { duplex. }\end{array}$ \\
\hline Channel coding & Turbo code \\
\hline Mobility & $350 \mathrm{~km} / \mathrm{h}$ \\
\hline Channel bandwidth & $1.4,3,5,10,15,20$ \\
\hline Modulation schemes & $\begin{array}{l}\text { Uplink: QPSK, } \\
\text { 16QAM,64QAM (optional) } \\
\text { Downlink: QPSK, 16QAM, } \\
\text { 64QAM }\end{array}$ \\
\hline Multiple access scheme & $\begin{array}{l}\text { Uplink: single carrier } \\
\text { frequency division multiple } \\
\text { access supports 50mbps } \\
\text { Downlink: OFDMA } \\
\text { supports } 100 \mathrm{mbps}\end{array}$ \\
\hline Peak data rate in LTE & $\begin{array}{l}\text { Uplink: } 75 \mathrm{mbps}(20 \mathrm{MHZ} \\
\text { bandwidth) } \\
\text { Downlink: } 150 \mathrm{mbps}(20 \\
\text { MHZ bandwidth) } \\
\text { Downlink: } 300 \mathrm{mbps}(20 \\
\text { MHZ bandwidth) }\end{array}$ \\
\hline $\begin{array}{l}\text { Multiple inputs multiple } \\
\text { outputs (MIMO) }\end{array}$ & $\begin{array}{l}\text { Uplink: } 1 \times 2,1 \times 4 \\
\text { Downlink: } 2 \times 2,4 \times 2,4 \times 4\end{array}$ \\
\hline Coverage & $\begin{array}{l}5-100 \mathrm{~km} \text { with minor } \\
\text { degradation after } 30 \mathrm{~km}\end{array}$ \\
\hline Latency & End-user latency $<10 \mathrm{mS}$ \\
\hline Quality of Service & $\begin{array}{l}\text { End-to-end Quality of } \\
\text { service permitting ordering } \\
\text { of different class of service. }\end{array}$ \\
\hline
\end{tabular}

Irrespective of advantageous communication characteristics of LTE, it is also associated with following challenges:

- Spectrum Harmonization: there are unclear approaches for LTE harmonization and distribution. The industry should address this type of problems in active participation, or else seamless roaming will remain just a visualization LTE network.

- Backhaul: the network operators face problems in adding raw capacity and understanding about full mesh backhaul when it originates to LTE backhaul.

- Security: the main aim to get the security procedure and compatible technology and make sure that the user only accesses the produced information. The information should be protected. It is seen that the security features and approaches can be prolonged and increased as compulsory by new services and threats. To make sure that the functions of security are compatible with worldwide accessibility.

- Handoff delay: handoff delay another significant Quality of Service related problems in LTE wireless network. In handoff delay procedure, the user understands the important drop in Quality of Service that will effect on the applications and upper-layer protocols.

- Less battery backup in LTE supported devices: in LTE supported devices due to the existence of a large number of receivers and transmitters, the battery consume high energy, and the machine shut down quickly. If want to increase the battery life in designing then the backup would enhance but as well as the size of devices would also increase.

- Integration of IP devices: to improve optimal facilities with higher bandwidth and high data rates, LTE technologies proposed the combination of IP and non-IP devices. This novel feature will integrate the infrastructure of entire current networks and also it will be smooth for users to access applications and services of the irrespective environment.

- $\quad$ Cost Affordability and Managing Accounts of Users: in LTE networks, managing user accounts has come to be complicated. The billing system is incapable of accomplished and figured out because of constant interaction of service providers and heterogeneity of LTE network. In terms of cost affordability, maximum problems consider that certain return degree of risks.

- Access Technologies: LTE network is a heterogeneous wireless environment that contains the number of radio technologies and also has overlay radio coverage. Allocating with different access technology is complex to the design of LTE network.

- A large Number of Operators: it difficult to find out that who is responsible for the delivery decision and how services can be protected in delivery because of a huge number of network operators. So it will be difficult by multipurpose trust relationship between operators.

- Congestion Control: congestion control is also a severe problem in LTE network. The prevention approach will necessitate the system to implement the scheduling approaches and admission control properly.

The next section discusses the existing approaches used to address the scheduling problems in LTE.

\section{RESEARCH TOWARDS SCHEDULING PROBLEMS}

This section discusses the existing research-based approaches towards enhancing the scheduling process in LTE-based Networks 
A scheduling plan in a satellite of LTE networks is presented by Aiyetoro et al. [23] to achieve the ideal QoS of LTE standards in respect of throughput and delay constraints. The authors have used a non-linear optimization approach in their scheme and also compared their presented approach with another optimal scheduling method. The outcomes of this study showed that the submitted plan achieves good throughput and gives optimal scheduling performance. Chen and Liu [24] have discussed a scheduling model for media streaming transmission in regards to providing play-out continuity over LTE services. The proposed model is based on discontinuous reception called as DRX and playout buffer with including three methods to solve this problem that is 1).remaining playout time, 2). Resource allocation technique and 3). Buffer level calculation technique. The presented model is tested on the NS3 simulator and reveals that it gives playout continuity and also reduces the energy cost of user equipment (UEs) at the low level and it is also shown that performance of the presented model is $50 \%$ better when compared with existing scheme. Fan et al. [25] have concentrated on the multiuser scheduling and pairing system with including inter and intracell interference in the LTE uplink cellular networks. The authors have constructed collaborative optimization problem by considering signal-to-interference-plusnoise ratio known (SINR) into single carrier frequency division multiple access (SC-FDMA), and frequency domain minimum mean- square error (FD-MMSE) in the concept of extending throughput of the overall network. The authors had divide optimization problem into two sub-problem and developed an algorithm to reduce the complexity of the optimization process. The study outcomes display that the presented method decreased the interference and performed well in compared to the existing plan.

Ferdosian et al. [26] have addressed the difficulty in scheduling for heterogeneous traffic in overload phase in LTE download link where user's channel details are varying and is not fixed. The authors had the analytical framework and presented a Fractional Knapsack algorithm to provide the QoS requirement and for efficient consumption of a resource for heterogeneous traffic in overload state of the cell. The result showed that the discussed approach gives suitable fairness and full-fill QoS requirements.

Ghavimi et al. [27] have focused on the issue related to the power allocation, Resource block (RB) and QoS requirement for M2M communication in uplink SC-FDMA dependent LTE-A networks. The authors have considered these problems as Binary Integer Programming (BIP) and based on Lagrange duality theory they split BIP problem into two subproblems. Afterward, the authors formulate computational algorithm based on Lagrange multiplier concept. The outcomes of the study show that the presented method defeats the existing Greedy algorithm in respect of providing maximum throughput and meets the QoS requirements. Grondalen et al.[28] has offered scheduler performance analysis parameter of different-different LTE downlinks channels with flat and frequency selective channels, saturated UDP and TCP traffic source and also considering the frequency-domain and time-domain schedulers. The analytical result shows that the presented approach achieves better tradeoff among the cell capacity and fairness for UDP and TCP traffic source. Guo et al. [29] have focused on multi-user multiple-input multiple outputs (MIMO) scheduling in LTE downlink cellular networks. The authors have constructed a framework in multiuser MIMO system in the presence of inter-stream interference and inter-user interference and developed an optimization problem approach based on pre-coding matrix indicator selection, joint user pairing, and RB allocation and considering these parameter optimal scheduling algorithm is elaborated which used to increase the throughput of the overall system. The simulated results display that it can achieve good performance.

Ko et al. [30] have designed scheme in which channel selection and frame scheduling taken as joint optimization problem approach which brings maximum throughput in LTE and also balancing the fairness among the WLAN and LTE. The authors have formulated another heuristic algorithm named as sequential operational algorithm (SOA) to reduce the complexity of The authors discovered approach, and in SOA the channels are selected according to channel ideal state probability, and LTE transmission interval can be calculated by fairness between the LTE and WLAN. The outcomes of this study are displayed that the presented method gets comparable throughput to optimal solution and also get fairness between LTE and WLAN. Li et al. [31] have introduced with an overview of MIMO techniques and multi-cell coordinate scheduling technique for LTE cellular networks. The authors have considered various approaches on SU-MIMO and multi-user MIMO for improving more performance parameter and discussed minimization of inter-cell interference methods for uplink and downlink in LTE. The study reveals that many aspects are discussed in terms of improving performance parameter. Lien et al.[32] has concentrated on Licensed-assisted access (LLA) collocated with wireless networks in which the authors developed optimum radio access which display the dynamic switch between scheduling dependent radio access and a random access plans. The result of this study shows the most favorable design of LLA networks.

Liu and Chen [33] have developed a framework based on downlink scheduling for enhancing and handle several complexities of media communication transmission using LTE network services by considering the following control module such as transmission control module and smart resource allocation module. The study outcomes show that discussed approach gives suitable scheduling performance for multimedia transmission over LTE networks. Luo et al. [34] have offered a new scheme which involved component carrier on/off scheduling and resource allocation. The presented system is based on the Lyapunov optimization to enhance the activity of packet throughput and uses Vapnik-Chervonenkis theoretical concept to determine the variation between predicted optimal value and practical value. The simulate outcomes of study generate good trade-off among the power utilization and packet throughput at the time of low traffic load. Mehaseb et al. [35] have studied on various uplink scheduling methods in LTE in regards to M2M communication for providing QoS requirements, power efficiency, scalability, and multi-hop connectivity. The outcome this study shows that author has tried to flash some light on the construction and designing requirement of scheduling techniques for $\mathrm{M} 2 \mathrm{M}$ communication to meet the goal of QoS, throughput and delay constraint. Minelli et al. [36] have reported the performance parameter of two conventional scheduler methods that are Proportional Fair (PF) and Round Robin (RR) for relay-enhanced LTE-A cellular networks. The authors have formulated the optimal model for extending the throughput and designed sub-optimal relay nodes placement for comparing RR and PF. The outcomes of the study show that $\mathrm{PF}$ is not performed well with respect to RR in several schemes.

Mostafa and Gadallah [37] have presented scheduling methodology to analyze the important factor of information in the data to be sent by machine type communications devices. (MTCDs).A statistical priority approach is used as the scheduling algorithm to define the scarce radio resources to MTCDs. The results offer that the presented method achieves great performance than other baseline schedulers in such a way 
that it having the least number of deadline misses for critical data packets. Pan et al. [38], has carried out to work on improving the network traffic and enhance the network capability for LTE-advanced (LTE-A) networks. A novel Downlink Traffic Arrangement approach is introduced to adopt arrangement downlink traffic with double connectivity improvement for LTE-A small network. The simulation outcomes show that novel scheduling approach can successfully improve the throughput of the system with double connectivity enhancement for LTE-A small network. The paper has presented by Ragaleux et al. [39] discuss the frequency and time resulting issues in LTE-A network. To resolve these issues, introduced an approach is Robust and Probable Conscious Scheduling, which protect an extensive range of scheduling objectives. The proposed method developed by three algorithms as follows:

i. For problem reduction.

ii. For First distribution and Users selection.

iii. For potential-conscious allocation.

The suggested scheduling approach is more effective than traditional methods in terms of satisfied operators in performance calculation outcomes. In the same way, Opportunistic Packet Delay Outage Ratio Aware function exceed the Proportional fair/MaxSNR effectiveness function for the multimedia scenario. Wang and Tsai [40], has discussed less system performance and lesser operator revenue problems arise in LTE network. In this study, authors have addressed a framework model is Pricing-conscious Resource Scheduling to resolve this conflict. It contains pricing and arrangement both module. Without loss of generalization, the framework model categorizes into three levels:

\section{- $\quad$ Golden (high)}

- $\quad$ Silver (medium)

- $\quad$ Bronze (low)

The result shows that the framework model makes an optimal balance between revenue and performance. It also supports Quality of Services for video service and Voice over Internet Protocol. Zolfaghari and Taheri [41], has addressed a non-real time flows issues. To tackle this issue here, authors introduced a novel scheduling approach is a Queue-conscious Channeladapted method in performance enhancement. It is well-matched with congestion control algorithms like minimal cost flow control algorithm, which considered on fluid flow statement. By the using proposed approach provide higher performance as compared to plain scheduling methods. The outcomes show the proposed scheduling technique with minimal cost flow control achieved high throughput and improved the elastic traffic. The study of Zolfaghari and Taheri [42], addressed issues in joint arrangement and congestion control optimization in LTE network. To improve these issues in LTE network explained about the scheduling methods which is Price Channel Quality. The outcomes display an optimal adjustment between the entire throughput and the equality for the joint algorithm, where scheduling and congestion control distinctly designed and executed without any support. In the study of Masegosa and Gzalvez [43], has introduced the first appraisal of the operation and performance of LTE vehicle-to-vehicle mode four communication inaccurate traffic conditions. In this study, the author has added a novel technique semi-persistent scheduling based on sense. It analyzed and measured the different types of transmission errors. The result shows the errors below 5\% for all outcomes. The proposed technique provides reduction with the distance between receiver and transmitter and the channel load.
In the study of Chang et al. [44] discussed the issues low throughput and high packet loss rate, which arises in LTE-A. Has introduced a technique which is based on cross-layer packet scheduling, and it is classified into two mechanisms:

i. Adaptive reward priority scheduling in MAC layer.

ii. Dynamic resource scheduling algorithm in the physical layer.

The technique provides the collaboration with the data symbol scheduling and Channel Quality Indicator at the physical layer. The outcomes show given technique offer an optimal solution for higher throughput and low packet delay in LTE-A network system. Kim and Park [45] have carried out the work on developing a framework integrated modeling based on two dimensional Markov chain model for the probability distribution of the number of arranged resource blocks. The outcomes defined that the presented framework model is reliable with spontaneous speculations. But it is tough to evaluate or examine the number of arranged resource blocks exactly. The study of Chakrapani [46] has described an efficient resource scheduling framework model, which is collaborating with narrowband internet things and improved machine type communication and also using two algorithmic approaches. The proposed technique reduces the complexity of limited bandwidth and enhances the coverage LTE network. The outcomes show that algorithmic approaches provide important profits increase to $80 \%$ over a loop based scheduling techniques and also the presented algorithmic approach is generic and appropriate to enhanced narrowband and user equipment implementation. The study carried out by Feng et al. [47] has addressed the scheduling algorithms and Quality of Services based LTE downlink scheduler for improving low communication and application information in smart grid communication. The simulation outcomes display the improvement of packet loss rate, delay, and average throughput. It is an important enhancement in an intelligent grid which based on LTE network. Alaa et al. [48] carried out the work on a machine to machine delay sensitive and lack of standardization problems in LTE network system. So authors have presented a technique a dynamic access grant time interval control scheduling approach which is based on the nonpreemptive queuing framework. The introduced scheduling approach has achieved an optimal percentage in reducing delay and as well as packet loss decreases in less percentage. Lau et al. [49], has introduced a framework model for inefficient radio resource usage in LTE broadcasting. The framework which defined by an author is Audience Driven Live Television Scheduling method, to enhance radio resource usage for television broadcasting over LTE network system. The proposed framework method usage three algorithms:
i. For television channels popularity calculation
ii. For resource approximation.
iii. For resource allocation.

The framework method is structured for both rural and suburban areas, and it is easy to relocate to different locations. The outcomes show that the proposed method attains higher broadcast proficiency and important conserved amount of radio resources. Cano et al. [50], has described the issue of low throughput and high delay cost of heterogeneity. The method is introduced by authors are Listen before Talk with Load Based Equipment (LT/LBE) and Carrier Sensing and Adaptive Transmission (CSAT) approach. The experimental outcomes show the heterogeneity cost is more for CSAT, and throughput of LTE is less when compared to the use of LT/LBE. In defective inter-technology detection, the consumption of explicit 
communication is more difficult in CSAT due to a higher possibility of loss compared to LT/LBE. Eladham et al. [51], has discussed lower system performance in-forms of spectral proficiency and lower throughput issues. For this issues introduced an algorithmic design of Improved Capacity Superior Proportional Fair downlink scheduling methods for achieving optimal system performance. It helps to enhance the heterogeneous network performance that allocates resource blocks to extend the system capacity and high fairness. The outcomes of this study show that proposed algorithm improves spectral proficiency and fairness as compared to other traditional approaches. Similarly, Xitlaly et al. [52] have also addressed scheduling problems. Hence, all the techniques mentioned above offer solutions towards scheduling problems. Table 2 summarizes the effectiveness of existing approaches towards scheduling in LTE-based networks.

Table.2. Summary of Existing Approaches

\begin{tabular}{|c|c|c|c|}
\hline Authors & Methods & Advantages & Limitations \\
\hline Aiyetoro et al.[23] & $\begin{array}{l}\text { Non-linear optimization } \\
\text { approach }\end{array}$ & $\begin{array}{l}\text { Ideal throughput and supports both } \\
\text { single and multiple traffic state. }\end{array}$ & $\begin{array}{l}\text { Only achieves maximum } \\
\text { throughput. }\end{array}$ \\
\hline Chen and Liu [24] & $\begin{array}{l}\text { Playout buffer and DRX based } \\
\text { mechanism. }\end{array}$ & $\begin{array}{l}\text { Playout continuity in steaming and } \\
\text { saves UEs energy. }\end{array}$ & $\begin{array}{l}\text { Insufficient to keep Quality of } \\
\text { experience of the client. Or } \\
\text { limited power saving. }\end{array}$ \\
\hline Fan et al. [25] & Optimization Algorithm & $\begin{array}{l}\text { Improved performance in LTE uplink } \\
\text { cellular networks. }\end{array}$ & No benchmarking \\
\hline Ferdosian et al.[26] & $\begin{array}{l}\text { Analytical framework and } \\
\text { Fractional Knapsack algorithm. }\end{array}$ & $\begin{array}{l}\text { Maximum fairness and balanced QoS } \\
\text { including throughput and delay. }\end{array}$ & $\begin{array}{l}\text { Less focus on practical } \\
\text { implementation }\end{array}$ \\
\hline Ghavimi et al. [27] & $\begin{array}{l}\text { Binary integer programming and } \\
\text { Lagrange concept. }\end{array}$ & Maximum throughput and less delay. & $\begin{array}{l}\text { Only applicable for uplink } \\
\text { transmission }\end{array}$ \\
\hline $\begin{array}{l}\text { Grondalen et al. } \\
{[28]}\end{array}$ & $\begin{array}{l}\text { Performance parameter analysis } \\
\text { approach }\end{array}$ & $\begin{array}{l}\text { Better tradeoff between cell capacity } \\
\text { and good fairness for TCP and UDP. }\end{array}$ & Results not benchmarked. \\
\hline Guo et al. [29] & Optimal scheduling algorithm. & $\begin{array}{l}\text { Achieves better throughput for all } \\
\text { network system. }\end{array}$ & Complexity not studied \\
\hline Ko et al. [30] & Sequential operation algorithm & $\begin{array}{l}\text { Get good throughput and good fairness } \\
\text { between LTE and WLAN. }\end{array}$ & Results not benchmarked. \\
\hline Lien and Liang [31] & Optimum radio access & $\begin{array}{l}\text { Suggest optimum design for LLA } \\
\text { networks. }\end{array}$ & Less exhaustive investigation \\
\hline Liu et al. [32] & Analytical modeling, hand-off & Efficient resource utilization & Complexity not studied \\
\hline Luo et al.[33] & Learning-based & Energy efficient & Narrowed test-environment \\
\hline Mehaseb et al. [34] & Energy-efficient modeling & Support multi-hop & Results not benchmarked. \\
\hline Minelli et al.[35] & Statistical modeling & Supports multiple traffic system & $\begin{array}{l}\text { Results not benchmarked., } \\
\text { includes overhead }\end{array}$ \\
\hline Mostafa et al. [36] & Statistical modeling & Supports various traffic system & $\begin{array}{l}\text { Results not benchmarked., } \\
\text { includes overhead }\end{array}$ \\
\hline Pan et al.[37] & $\begin{array}{l}\text { Analytical modeling, splitting } \\
\text { approach }\end{array}$ & Enhanced network throughput & Restricted to small cell only \\
\hline Pan et al. [38] & Downlink Traffic Arrangement & $\begin{array}{l}\text { Increase throughput, double } \\
\text { connectivity }\end{array}$ & No benchmarking, high delay \\
\hline Ragaleux et al. [39] & $\begin{array}{l}\text { Robust and Probable Conscious } \\
\text { Scheduling }\end{array}$ & Low packet delay & $\begin{array}{l}\text { Doesn't emphasize on device } \\
\text { heterogeneity }\end{array}$ \\
\hline Wang and Tsai [40] & $\begin{array}{l}\text { Pricing-conscious Resource } \\
\text { Scheduling }\end{array}$ & $\begin{array}{l}\text { High throughput for voice and video } \\
\text { service over the internet }\end{array}$ & No benchmarking \\
\hline $\begin{array}{l}\text { Zolfaghari and } \\
\text { Taheri [41] }\end{array}$ & $\begin{array}{l}\text { Queue-conscious Channel- } \\
\text { adapted method }\end{array}$ & High throughput, traffic control. & No benchmarking \\
\hline Zolfaghari and & Price Channel Quality & Optimal throughput, designing, and & Outcomes not compared with \\
\hline
\end{tabular}




\begin{tabular}{|c|c|c|c|}
\hline Taheri [42] & scheduling method & implementation without any support & any existing system \\
\hline $\begin{array}{l}\text { Masegosa and } \\
\text { Gzalvez [43] }\end{array}$ & semi-persistent scheduling & Simplified Technique & $\begin{array}{l}\text { Will lead to complexity and } \\
\text { overhead for large network }\end{array}$ \\
\hline Chang et al. [44] & $\begin{array}{l}\text { Adaptive reward priority and } \\
\text { Dynamic resource scheduling }\end{array}$ & Low packet delay, higher throughput. & Lack of benchmarking \\
\hline Kim and Park [45] & $\begin{array}{l}\text { An integrated modeling based on } \\
\text { 2D Markov chain model. }\end{array}$ & $\begin{array}{l}\text { Reliability with spontaneous } \\
\text { speculations. }\end{array}$ & $\begin{array}{l}\text { Less effective on analyzing } \\
\text { RBs. }\end{array}$ \\
\hline Chakrapani [46] & Algorithmic approach & $\begin{array}{l}\text { Profits increase to } 80 \% \text { over a loop } \\
\text { based scheduling. }\end{array}$ & Narrowed test environment \\
\hline Feng et al. [47] & $\begin{array}{l}\text { Quality of Services based LTE } \\
\text { downlink scheduler }\end{array}$ & $\begin{array}{l}\text { Average delay and Packet loss rate, } \\
\text { high throughput. }\end{array}$ & $\begin{array}{l}\text { Focuses only on downlink } \\
\text { transmission }\end{array}$ \\
\hline Alaa et al. [48] & $\begin{array}{l}\text { dynamic access grant time } \\
\text { interval control scheduling }\end{array}$ & $\begin{array}{l}\text { Reduce delay and the low percentage of } \\
\text { packet loss. }\end{array}$ & $\begin{array}{l}\text { Performance depends on the } \\
\text { hypothetical threshold }\end{array}$ \\
\hline Lau et al. [49] & $\begin{array}{l}\text { Audience-Driven Live } \\
\text { Television Scheduling }\end{array}$ & $\begin{array}{l}\text { Easy to relocate to different places, } \\
\text { higher broadcast efficiency. }\end{array}$ & Lack of benchmarking \\
\hline Cano et al. [50] & Analytical approach & Considers heterogeneity factor & $\begin{array}{l}\text { Leads to complexity in the } \\
\text { longer run }\end{array}$ \\
\hline Eladham et al. [51] & Downlink scheduling & $\begin{array}{l}\text { Studies both homogeneous \& } \\
\text { heterogeneous network }\end{array}$ & Model validation missing \\
\hline Xitlaly et al. [52] & Propagation model, round robin & $\begin{array}{l}\text { The simplified approach, better } \\
\text { throughput }\end{array}$ & Narrowed test environment \\
\hline
\end{tabular}

\section{RESEARCH GAP}

After reviewing the existing approaches to scheduling, it can be observed that all the methods have significant advantages as well as some specific limitation. However, apart from there are some set of problems that were existing during the implementation process of existing techniques but they were not reported to be addressed. This section identifies such forms of research gaps and briefs about it. Following are the prominent research gaps that are obtained after reviewing the existing approaches of scheduling in TE-based networks.

- OFDM Problems Deemed Low Importance: A closer look into the existing system shows that consideration of OFDM has been less utilized after 3G-based network whereas in reality, OFDM is highly essential for downlink transmission in LTE while the uplink transmission in LTE uses another mechanism (i.e., SC-FDMA). Adoption of OFDM in LTE degrades the scheduling decision in the presence of largescale and dynamic traffic situation, which requires immediate attention.

- Discrete Perspective of Delay: Irrespective of a different number of research work addressing delay problem in the LTE-based network, it has been observed that such research work has not considered the complexity in characterizing network delay as such network delay originated from the dynamic queues encounters a potential challenge in the design phase. It is because they cannot be realized using frequently utilized Markov chain. Such problem takes challenging shape when subjected to error-prone channels with multiple numbers of users in it.

- Lack of Complexity Analysis: The existing approaches towards scheduling LTE-based network have analyzed its effectiveness only on the basis of performance parameters, e.g., throughput, $\mathrm{CDF}$, delay, energy, etc. However, there is no such evidence if such techniques can be practically executed in the real-time scenario as it lacks any form of complexity analysis.

- Lower Emphasis to Queue Management: A queue always exists between server and users, which are less likely to be seen to be discussed in existing literature. Existing default queue management system only considers delay associated with one targeted server ignoring the fact that there are many another server that is also connected to it. Such mechanism of allocation of server eventually disrupts scheduling performance.

- Less Significance towards HARQ: There is various literature published in past decade discussing the benefits of using Hybrid Automatic Repeat Request for facilitating the reliability of transmission in wireless networks. It does so by harnessing the errors occurring during transmission. The mechanism of HARQ scheme by using feedbacks assists the node to take the much better decision of scheduling. However, irrespective of its beneficial features, HARQ is not found to be investigating in connection with the scheduling practices in existing research community towards LTE-based networks.

- Lack of Benchmarked Model: The existing research-based approaches towards improving communication performance in LTE are highly scattered, and less work towards scheduling has been carried out. However, the existing amount of studies towards scheduling are not reported to be benchmarked or standardized that reduces the possibility of practical utilization until further research is continued. The inclusion of exhaustive traffic model, test scenarios in LTE are less considered in existing scheduling approaches. 
The above-stated research gap is found and considered to be addressed in the existing system.

\section{CONCLUSION \& FUTURE WORK}

The prime intention of the proposed study was to study the existing research work being carried out towards leveraging the scheduling process in LTE-based networks. It has been noticed that LTE uses two different access schemes explicitly for uplink (OFDM/OFDMA) as well as for downlink (SC-FDMA) transmission. The paper has discussed the theoretical significance of LTE followed by existing research-based solutions. Although there have been various studies carried out towards scheduling techniques in LTE, still there are open-end problems. Almost all the existing approaches are associated with limitations. It was also observed that there is a rising level of interest towards improving the performance of throughput and delay; however, existing studies are not found to offer any such robust solution for this. While there exist various techniques but very few of them are found to address the practicality of implementation, which is the major flaw and set back of the majority of existing approaches. Hence, this paper contributes to i) offer a quick snapshot of the LTE-based network, ii) compact discussion of some recent implementation work towards scheduling, iii) identifying the advantage and limitations associated with each implementation, and iv) discussion the open -end problems regarding research gap.

The future work will be definitely towards bridging the research gap being identified in this paper. The first approach will be to develop a model that offers to schedule for improving both throughput and delay performance at the same time considering all sorts of practical constraints in the LTE-based network. The second possible future work could be to include the hybrid ARQ mechanism to boost up the channel efficiency of LTE, which will indirectly improve the communication performance too.

\section{REFERENCES}

[1] B. Furht, Syed A. Ahson, Long Term Evolution: 3GPP LTE Radio and Cellular Technology, 2016

[2] H. Zhang, Sami Khairy, Lin X. Cai, Zhu Han, Resource Allocation in Unlicensed Long Term Evolution HetNets, Springer, 2017

[3] S. Sesia, Matthew Baker, Issam Toufik, LTE - The UMTS Long Term Evolution: From Theory to Practice, John Wiley \& Sons, 2011

[4] A. Paradisi, Michel Daoud Yacoub, Fabrício Lira Figueiredo, Tania Tronco, Long Term Evolution: 4G and Beyond, Springer, 2015

[5] K. Pahlavan, Prashant Krishnamurthy, Principles of Wireless Access and Localization, John Wiley \& Sons, 2013

[6] A. Yahya, LTE-A Cellular Networks: Multi-hop Relay for Coverage, Capacity and Performance Enhancement, Springer, 2016

[7] Q-D Ho, Daniel Tweed, Tho Le-Ngoc, Long Term Evolution in Unlicensed Bands, Springer, 2016

[8] Panagopoulos, Athanasios D, Handbook of Research on Next Generation Mobile Communication Systems, IGI Global, 2015

[9] M. Rupp, Stefan Schwarz, Martin Taranetz, The Vienna LTE-Advanced Simulators: Up and Downlink, Link and System Level Simulation, Springer, 2016
[10] M. Danilo Abrignani, L. Giupponi, A. Lodi and R. Verdone, "Scheduling M2M traffic over LTE uplink of a dense small cells network," 2015 International Symposium on Wireless Communication Systems (ISWCS), Brussels, 2015, pp. 760-764.

[11] R. R. Su and I. S. Hwang, "Efficient resource allocation scheme with grey relational analysis for the uplink scheduling of 3GPP LTE networks," 2016 IEEE International Conference on Industrial Technology (ICIT), Taipei, 2016, pp. 599-603

[12] J. Park and Jaemin Ahn, "Improving frequency multiplexing of random access resources for TDD system enhancement," 2016 Eighth International Conference on Ubiquitous and Future Networks (ICUFN), Vienna, 2016, pp. 661-666.

[13] M. Ayhan, L. Zappaterra and H. A. Choi, "Geometric average-based fast-converging proportional fair scheduling for LTE downlink transmissions," 2016 1st International Workshop on Link- and System Level Simulations (IWSLS), Vienna, 2016, pp. 1-6.

[14] V. C. Gungor and O. G. Uyan, "QoS-aware downlink scheduling algorithm for LTE networks: A case study on edge users," 2017 25th Signal Processing and Communications Applications Conference (SIU), Antalya, 2017, pp. 1-4.

[15] H. Shan, Y. Zhang, W. Zhuang, A. Huang, and Z. Zhang, "User Behavior-Aware Scheduling Based on TimeFrequency Resource Conversion," in IEEE Transactions on Vehicular Technology, vol. 66, no. 9, pp. 8429-8444, Sept. 2017.

[16] H. A. M. Ramli, M. K. Hasan, A. F. Ismail, A. H. Abdalla and K. Abdullah, "An investigation of packet scheduling algorithms for Long Term Evolution-Advanced," 2012 IEEE Conference on Open Systems, Kuala Lumpur, 2012, pp. 1-5.

[17] H. W. Tseng, W. C. Lee, C. R. Sheu, Y. H. Lee and Y. G. Jan, "Applying genetic algorithms to the data traffic scheduling and performance analysis of a long-term evolution system," 2012 International Symposium on Intelligent Signal Processing and Communications Systems, New Taipei, 2012, pp. 183-188.

[18] O. Ergul, O. Yilmaz, A. T. Koc and O. B. Akan, "DRX and QoS-aware energy-efficient uplink scheduling for long term evolution," 2013 IEEE Global Communications Conference (GLOBECOM), Atlanta, GA, 2013, pp. 4644-4649

[19] A. D. Maleki and B. Abolhassani, "New scheduling scheme for green communications in long term evolution networks," in IET Communications, vol. 8, no. 14, pp. 2438-2444, Sept. 25, 2014

[20] J. G. Neto, F. A. C. M. Cardoso, P. A. V. Ferreira and M. H. M. Costa, "Customizable algorithm for data scheduling in long term evolution networks," 2015 Information Theory and Applications Workshop (ITA), San Diego, CA, 2015, pp. 123-129.

[21] S.-B. Lee, S. Choudhury, A. Khoshnevis, S. Xu, and S. Lu, "Downlink MIMO with frequency-domain packet scheduling for 3GPP LTE," in Proceedings of the 28th IEEE Conference on Computer Communications, pp. 2611-2615, 2009 
[22] N. Wei, A. Pokhariyal, T. B. Sørensen, T. E. Kolding, and P. E.Mogensen, "Performance of MIMO with frequency domain packet scheduling in UTRAN LTE downlink," in Proceedings of the IEEE Vehicular Technology Conference (VTC '07), pp. 1177-1181, April 2007.

[23] G. Aiyetoro, F. Takawira and T. Walingo, "Near-optimal packet scheduling scheme in satellite LTE networks," in IET Communications, vol. 11, no. 15, pp. 2311-2319, 10 192017.

[24] Y. Chen and G. Liu, "Playout buffer and DRX aware scheduling scheme for video streaming over LTE system," in IET Communications, vol. 10, no. 15, pp. 1971-1978, 10 132016.

[25] J. Fan, D. Lee, G. Y. Li and L. Li, "Multiuser Scheduling and Pairing With Interference Mitigation for LTE Uplink Cellular Networks," in IEEE Transactions on Vehicular Technology, vol. 64, no. 2, pp. 481-492, Feb. 2015.

[26] N. Ferdosian, M. Othman, B. M. Ali and K. Yeah Lun, "Multi-Targeted Downlink Scheduling for Overload-States in LTE Networks: Proportional Fractional Knapsack Algorithm With Gaussian Weights," in IEEE Access, vol. 5, pp. 3016-3027, 2017.

[27] F. Ghavimi, Y. W. Lu and H. H. Chen, "Uplink Scheduling and Power Allocation for M2M Communications in SCFDMA-Based LTE-A Networks With QoS Guarantees," in IEEE Transactions on Vehicular Technology, vol. 66, no. 7, pp. 6160-6170, July 2017.

[28] O. Grøndalen, A. Zanella, K. Mahmood, M. Carpin, J. Rasool and O. N. Østerbø, "Scheduling Policies in Time and Frequency Domains for LTE Downlink Channel: A Performance Comparison," in IEEE Transactions on Vehicular Technology, vol. 66, no. 4, pp. 3345-3360, April 2017.

[29] W. Guo, J. Fan, G. Y. Li, Q. Yin and X. Zhu, "Adaptive SU/MU-MIMO scheduling schemes for LTE-A downlink transmission," in IET Communications, vol. 11, no. 6, pp. 783-792, 4202017

[30] H. Ko, J. Lee and S. Pack, "Joint Optimization of Channel Selection and Frame Scheduling for Coexistence of LTE and WLAN," in IEEE Transactions on Vehicular Technology, vol. PP, no. 99, pp. 1-1.

[31] G. Y. Li, J. Niu, D. Lee, J. Fan and Y. Fu, "Multi-Cell Coordinated Scheduling and MIMO in LTE," in IEEE Communications Surveys \& Tutorials, vol. 16, no. 2, pp. 761-775, Second Quarter 2014

[32] S. Y. Lien, J. Lee and Y. C. Liang, "Random Access or Scheduling: Optimum LTE Licensed-Assisted Access to Unlicensed Spectrum," in IEEE Communications Letters, vol. 20, no. 3, pp. 590-593, March 2016.

[33] Q. Liu and C. W. Chen, "Smart Downlink Scheduling for Multimedia Streaming Over LTE Networks With Hard Handoff," in IEEE Transactions on Circuits and Systems for Video Technology, vol. 25, no. 11, pp. 1815-1829, Nov. 2015.

[34] Z. Luo, M. Ding and H. Luo, "CC On/Off Scheduling Using Learning-Based Prediction for LTE in the Unlicensed Spectrum," in IEEE Communications Letters, vol. 19 , no. 12, pp. 2158-2161, Dec. 2015.
[35] M. A. Mehaseb, Y. Gadallah, A. Elhamy and H. Elhennawy, "Classification of LTE Uplink Scheduling Techniques: An M2M Perspective," in IEEE Communications Surveys \& Tutorials, vol. 18, no. 2, pp. 1310-1335, Secondquarter 2016.

[36] M. Minelli, M. Ma, M. Coupechoux and P. Godlewski, "Scheduling Impact on the Performance of Relay-Enhanced LTE-A Networks," in IEEE Transactions on Vehicular Technology, vol. 65, no. 4, pp. 2496-2508, April 2016.

[37] A. E. Mostafa and Y. Gadallah, "A Statistical PriorityBased Scheduling Metric for M2M Communications in LTE Networks," in IEEE Access, vol. 5, pp. 8106-8117, 2017.

[38] M. S. Pan, T. M. Lin, C. Y. Chiu and C. Y. Wang, "Downlink Traffic Scheduling for LTE-A Small Cell Networks With Dual Connectivity Enhancement," in IEEE Communications Letters, vol. 20, no. 4, pp. 796-799, April 2016.

[39] A. Ragaleux, S. Baey and M. Karaca, "Standard-Compliant LTE-A Uplink Scheduling Scheme With Quality of Service," in IEEE Transactions on Vehicular Technology, vol. 66, no. 8, pp. 7207-7222, Aug. 2017.

[40] Y. C. Wang and T. Y. Tsai, "A Pricing-Aware Resource Scheduling Framework for LTE Networks," in IEEE/ACM Transactions on Networking, vol. 25, no. 3, pp. 1445-1458, June 2017.

[41] A. Zolfaghari and H. Taheri, "Queue-Aware ChannelAdapted Scheduling and Congestion Control for BestEffort Services in LTE Networks," in Canadian Journal of Electrical and Computer Engineering, vol. 38, no. 2, pp. 170-182, Spring 2015.

[42] A. Zolfaghari and H. Taheri, "Joint Best Price-CQI Product Scheduling and Congestion Control for LTE," in Canadian Journal of Electrical and Computer Engineering, vol. 39, no. 4, pp. 255-267, Fall 2016.

[43] R. Molina-Masegosa and J. Gozalvez, "System Level Evaluation of LTE-V2V Mode 4 Communications and Its Distributed Scheduling," 2017 IEEE 85th Vehicular Technology Conference (VTC Spring), Sydney, NSW, 2017, pp. 1-5.

[44] B. J. Chang, P. Y. Chang and Y. H. Liang, "Adaptive packet scheduling and radio RB allocation for LTE-A toward 5G relaying communications," 2017 8th IEEE Annual Information Technology, Electronics and Mobile Communication Conference (IEMCON), Vancouver, BC 2017, pp. 358-364.

[45] Y. Kim and S. Park, "Analytical Calculation of Spectrum Requirements for LTE-A Using the Probability Distribution on the Scheduled Resource Blocks," in IEEE Communications Letters, vol. PP, no. 99, pp. 1-1.

[46] A. Chakrapani, "Efficient resource scheduling for eMTC/NB-IoT communications in LTE Rel. 13," 2017 IEEE Conference on Standards for Communications and Networking (CSCN), Helsinki, 2017, pp. 66-71.

[47] F. Feng, F. Peng, B. Yan, S. Lin and J. Zhang, "QoS-based LTE downlink scheduling algorithm for smart grid communication," 2017 IEEE 9th International Conference on Communication Software and Networks (ICCSN), Guangzhou, 2017, pp. 548-552. 
[48] Y. Alaa, H. M. ElAttar, F. Digham, L. H. Afify and H. Elbadawy, "LTE dynamic scheduling scheme for massive M2M and H2H communication," 2017 IEEE 8th Annual Ubiquitous Computing, Electronics and Mobile Communication Conference (UEMCON), New York City, NY, 2017, pp. 478-482.

[49] C. P. Lau, A. Alabbasi and B. Shihada, "An Efficient Live TV Scheduling System for 4G LTE Broadcast," in IEEE Systems Journal, vol. 11, no. 4, pp. 2737-2748, Dec. 2017.

[50] C. Cano, D. J. Leith, A. Garcia-Saavedra and P. Serrano, "Fair Coexistence of Scheduled and Random Access Wireless Networks: Unlicensed LTE/WiFi," in IEEE/ACM
Transactions on Networking, vol. 25, no. 6, pp. 3267-3281, Dec. 2017.

[51] A. S. Eladham, N. ElShennawy and A. Sarhan, "Modified capacity enhanced PF (MCE-PF) downlink scheduling algorithm for heterogeneous LTE-Advanced Networks," 2017 13th International Computer Engineering Conference (ICENCO), Cairo, Egypt, 2017, pp. 1-7.

[52] R. O. Xitlaly, G. R. J. María and V. C. Norman, "Analysis of the throughput performance for a LTE-A network in Managua using the strategies of the Packet Scheduling and frequency bands 1, 2 and 3 FDD," 2017 IEEE 37th Central America and Panama Convention (CONCAPAN XXXVII), Managua, Nicaragua, 2017, pp. 1-6. 